\title{
The interplay between HIF-1 $\alpha$ and long noncoding GAS5 regulates the JAK1/STAT3 signalling pathway in hypoxia-induced injury in myocardial cells
}

\author{
Yanwei $\mathrm{Li}^{1}$, Bing Song ${ }^{2}$, Jinlei $\mathrm{Liu}^{3}$, Yuqiang $\mathrm{Li}^{4}$, Jiebing Wang ${ }^{5}$, Na Liu ${ }^{6}$, Wei Cui ${ }^{7}$ \\ ${ }^{1}$ Management Center of Chronic Diseases, The First Affiliated Hospital of Jinzhou Medical University, Jinzhou, China; ${ }^{2}$ Department of Endocrine \\ and Metabolic Diseases, The First Affiliated Hospital of Jinzhou Medical University, Jinzhou, China; ${ }^{3}$ Department of Radiology, The First Affiliated \\ Hospital of Jinzhou Medical University, Jinzhou, China; ${ }^{4}$ Biobank Department, The First Affiliated Hospital of Jinzhou Medical University, Jinzhou, \\ China; ${ }^{5}$ Department of Ultrasonography, The First Affiliated Hospital of Jinzhou Medical University, Jinzhou, China; ${ }^{6}$ Endocrinology Department, \\ Affiliated Hospital of Weifang Medical College, Weifang, China; ${ }^{7}$ Liaoning Jinzhou Inspection and Testing Certification Center, Jinzhou, China \\ Contributions: (I) Conception and design: Y Li, W Cui; (II) Administrative support: W Cui, J Wang; (III) Provision of study materials or patients: \\ B Song, J Liu; (IV) Collection and assembly of data: Y Li, B Song; (V) Data analysis and interpretation: Y Li, B Song; (VI) Manuscript writing: All \\ authors; (VII) Final approval of manuscript: All authors. \\ Correspondence to: Wei Cui. Liaoning Jinzhou Inspection and Testing Certification Center, 39 Jiefang East Road, Linghe District, Jinzhou 121001, \\ China. Email: ss6866@aliyun.com; kong126721@163.com.
}

Background: Long non-coding RNA (lncRNA) GAS5 is associated with hypoxia-induced diseases whereas hypoxia-inducible factor- $1 \alpha(\mathrm{HIF}-1 \alpha)$ plays an important role in hypoxic injury of cells. The current study explores the regulatory functions of GAS5/HIF- $1 \alpha$ which co-play in anoxic injury among rat cardiomyocytes H9C2 cells.

Methods: Hypoxia in vitro model was established through anoxic incubation while normal culture of H9C2 cells was considered as control. The expression levels of GAS5 and HIF-1 $\alpha$ were quantified through RTqPCR. CCK-8 was applied to determine cell viability. Cell apoptosis rate was calculated using flow cytometry whereas inflammatory cytokines were detected using ELISA method. The impact of downregulating GAS5 or HIF-1 $\alpha$ or both upon hypoxic cells was assessed on the basis of changes in cell viability, apoptosis, and inflammatory response. The activity of JAK1/STAT3 signaling was evaluated through RT-qPCR for mRNA expression. AG490 was introduced to inactivate JAK1/STAT3 pathway and to unveil the impact of JAK1/ STAT3 signaling on GAS5/HIF-1 $\alpha$ and cell viability, apoptosis and inflammation in hypoxic cells.

Results: The results infer that hypoxia suppressed cell viability, promoted inflammation and apoptosis among H9C2 cells. GAS5 or HIF-1 $\alpha$ recorded higher expression in hypoxia-induced cells whereas the cell viability got restored with reduction in inflammation and apoptosis. The downregulation of HIF-1 $\alpha$ enhanced the protective effect of knocking down GAS5 in hypoxia H9C2 cells. JAK1/STAT3 signaling pathway got activated in hypoxic cells and was regulated by GAS5 and HIF-1 $\alpha$. The inhibition of signaling pathway increased the cell viability but it decreased both inflammation and apoptosis.

Conclusions: GAS5 and HIF-1 $\alpha$ could regulate hypoxic injury in H9C2 cells through JAK1/STAT3 signaling pathway. This scenario suggests that the inhibitors of GAS5 and HIF-1 $\alpha$ may synergize with AG490 to protect myocardial cells from hypoxic injury.

Keywords: Long non-coding RNA GAS5 (lncRNA GAS5); hypoxia; hypoxia-inducible factor-1 $\alpha$ (HIF-1 $\alpha$ ); JAK1/STAT3

Submitted Sep 09, 2020. Accepted for publication Nov 29, 2020.

doi: $10.21037 / \mathrm{cdt}-20-773$

View this article at: http://dx.doi.org/10.21037/cdt-20-773

^ ORCID: 0000-0001-6870-1258. 


\section{Introduction}

Cardiac muscle tissues are highly sensitive and prone to hypoxic environment. In the event of hypoxia, it can not only lead to heart failure after traumas, but also injure multiple organs resulting in multi-organ dysfunction (1). Various studies have been conducted so far focusing molecular regulators in myocardial cells (2). Hypoxia models are adopted in myocardial infarction research and are often used in evaluating the effects of active substances on cellular morphology and functions (3).

In the recent years, long non-coding RNAs (lncRNAs) emerged as influential modulators for various diseases including cancers. These RNAs mediate the disease phenotypes via interplay with other molecules in cells such as proteins, DNA and other RNAs. So these molecules can be considered as potential therapeutic targets (4). Further, lncRNAs were investigated in cardiovascular diseases as well. lncRNA MALAT1 is recognized as a cancer biomarker with abnormally high expression in many cancers. So this biomarker is regarded as a potential target in the treatment of cancer (5). LncRNA GAS5 has been identified as a tumor suppressor in few cancer types such as gastric cancer, prostate cancer, bladder cancer, etc. (6). In recent years, there is an increasing attention towards investing lncRNAs in hypoxia-induced myocardial cells (7). In myocardial ischemia-reperfusion in vitro model, GAS5 upregulation contributed to increase in the apoptosis rate of $\mathrm{H} 9 \mathrm{C} 2$ cells, by enhancing LAS1 via P38/MAPK signaling pathway (8). Recently, it was reported that GAS5 can be downregulated by a traditional Chinese medicine Astragaloside IV. This therapy results in reduced cell injury via $\mathrm{Pi} 3 \mathrm{k} / \mathrm{mTOR}$ signaling in H9C2 cells (9). Previously, the apoptosis of hypoxia-induced myocardial cells has been disclosed to have been adjusted by GAS5. But it is unclear whether GAS5 can modulate inflammation and cell viability in hypoxia-induced H9C2 cells.

Hypoxia-inducible factor-1 $\alpha$ (HIF- $1 \alpha$ ) has been frequently investigated in both in vitro and in vivo models of myocardial hypoxia. This factor can be stimulated by hypoxia treatment (10). Further, the inhibition of HIF$1 \alpha$ was found to alleviate apoptosis and inflammatory cytokine in glucose-induced cardiomyocyte injury model (11). In hypoxia-induced H9C2 cells, it has been inferred that Genistein could decrease H9C2 cell apoptosis from hypoxic injury through the inhibition of HIF-1 $\alpha$ (12). Although it is an established fact that GAS5 or HIF-1 $\alpha$ could independently function in myocardial hypoxia in vitro, no research conducted so far has correlated them in detail. Therefore, the authors of the current study hypothesized that GAS5 might co-regulate the cell functions with HIF-1 $\alpha$ in hypoxia-induced $\mathrm{H} 9 \mathrm{C} 2$ cells. The present study utilized physical methods to establish hypoxia in myocardial cells in in vitro model so as to execute an in-depth exploration into the functions of lncRNA GAS5/HIF-1 $\alpha$ among hypoxic H9C2 cells.

We present the study in accordance with the MADR reporting checklist (available at http://dx.doi.org/10.21037/ cdt-20-773).

\section{Methods}

\section{Ethic statement}

All the experiments were performed in this study in compliance with the guidelines of Institutional Ethical Review Board (IEC), The First Affiliated Hospital, Jinzhou Medical University, Jinzhou City, Liaoning Province, China.

\section{Cell culture and treatment}

Rat H9C2 cell line, originally from embryonic rat cardiac tissues, shares numerous features in common with primary cardiomyocytes (13). Compared to primary cardiomyocytes, derived from neonatal rats, $\mathrm{H} 9 \mathrm{C} 2$ cell line is easy to acquire. A lot of research investigations are ongoing using H9C2 to establish hypoxia-stimulated cellular model of myocardial infarction (14). Therefore, in this study, the authors used H9C2 cells to simulate primary cardiomyocytes in hypoxic conditions. H9C2 cell line was procured from ATCC (rat, CL-0089, US). The cells were first thawed and then incubated in DMEM (Gibco, US) with $100 \mathrm{U} / \mathrm{mol}$ penicillin and streptomycin in $37{ }^{\circ} \mathrm{C}$ under $5 \% \mathrm{CO}_{2}$. The cells were then cultured in incubator for 12 and 24 hours with $95 \%$ $\mathrm{N}_{2}$ and $5 \% \mathrm{CO}_{2}$ in $37{ }^{\circ} \mathrm{C}$ to induce anoxic injury and form hypoxia group of $\mathrm{H} 9 \mathrm{C} 2$ cells. The cells in control group were incubated for 24 hours in $37^{\circ} \mathrm{C}$, air and under $5 \% \mathrm{CO}_{2}$. Inverted microscope was used to observe cell morphology at 0,12 and $24 \mathrm{~h}$ at $\times 100$ and $\times 400$ magnifications (Olympus, Japan). The cells in hypoxia group and those cells in log phase in control group were selected for further experiments.

\section{Cell transfection and signaling inbibition}

The cells in hypoxia group (24 h) were selected to modulate 
the expression of GAS5 and HIF-1 $\alpha$ through transfection. $1.0 \times 10^{6}$ cells were cultured in $25 \mathrm{~cm}^{2}$ culture bottle (T25, Orange Scientific, Belgium) and then incubated with $8 \mathrm{~mL}$ of DMEM for one night (Beyotime, Shanghai, China). The culture was replaced with $900 \mu \mathrm{L}$ of Opti-MEM serumfree medium at $0.5 \mathrm{~h}$ before the transfection process was initiated (Thermofisher, CA, USA). Mixture A was prepared with $25 \mu \mathrm{L} /$ culture bottle of siGAS5, siHIF- $1 \alpha$ and si$\mathrm{NC}$ respectively and made up with Opti-MEM to the final volume of $500 \mu \mathrm{L}$ for each group. Mixture B was prepared with $8 \mu \mathrm{L}$ Lipofectamine 2000 Reagent (Invitrogen, USA) and was diluted by Opti-MEM to the final volume of $500 \mu \mathrm{L}$. After incubating mixture B for 5 mins, it was added into mixture A and blended gently. Then the blend was incubated for 20 mins at room temperature. Thereafter, the mixture was added into cell culture bottle in a dropwise fashion. After incubation with $5 \% \mathrm{CO}_{2}$ at $37{ }^{\circ} \mathrm{C}$ for $6 \mathrm{~h}$, the medium was changed with RPMI 1640 (Thermofisher, CA, USA). Sequences such as si-GAS5, si-HIF- $1 \alpha$ and siNC were synthesized at Tianjin Sier Biotech (Tianjin, China). The sequences are as below si-GAS5 (rat), Sense: 5 '-UAUAAAGGUACCACAUGUTT-3'; Antisense: 5 '-ACAUGUGGUACCUUUAUACTT-3'; si-HIF- $1 \alpha$ (rat), Sense: 5'-GAAACUCUUCCAAGC-AAUUTT-3'; Antisense: 5'-AAUUGCUUGGAAGAGUUUCTT-3'; NC (rat), Sense: 5'-UUCUCCGAACGUGUCACGUTT-3'; Antisense: 5'-ACGUGACACGUUCGGAGAATT-3'. After transfection, hypoxia cells were divided into subgroups such as hypoxia control group, si-NC, si-GAS5 and si-HIF$1 \alpha$ groups. The co-transfected groups were generated in the same way except a fact that mixture A was a blend of si$\mathrm{NC}$ and si-GAS5 or si-GAS5 and si-HIF-1 $\alpha$, which further divided into two groups i.e., si-GAS and si-GAS + si-HIF- $1 \alpha$.

\section{$A G-490$ treatment}

The cells in hypoxia group ( $24 \mathrm{~h})$ were selected and treated with $10 \mathrm{nM}$ of JAK1/STAT3 inhibitor AG-490 agent for one hour (HY-12000, MCE, Shanghai, China) to inactivate JAK1/STAT3 signaling pathway. Therefore, two subgroups were formed with different JAK1/STAT3 activities. The cells were prepared for further use.

\section{$R T-q P C R$}

The cells in different groups were selected to detect their expression levels. One $\mathrm{mL}$ of TRIzol reagent (Invitrogen, USA) was added into the medium to lyse the cells and extract total RNAs. The RNAs were reverse-transcribed to cDNAs by strict adherence to manufacturer's instructions. Five pmol/ $\mu \mathrm{L}$ PCR primers ( $1 \mu \mathrm{L}$ reverse and $1 \mu \mathrm{L}$ forward), from each gene, were mixed with cDNAs in each subgroup respectively using $2 \times$ SYBR Premix Ex Taq (Takara, Tokyo, Japan) and Sterile Double Distilled Water (Beyotime, Shanghai, China). Primer sequences are displayed herewith: GAS5: forward, 5'-CTTGCCTGGACCAGCTTAAT-3', reverse, 5'-GAAGCCGACTCTCCATACCT-3'; HIF$1 \alpha$ : forward, 5'-ACTGATTGCATCTCCACCTTCT-3', reverse, 5'-TCGCTTCCTCTGAGCATTCT-3'; JAK1: sense, 5'-CATGGTGGAAGAGTTTGTGGAA-3', antisense, 5'-CAGCTGTTTGGCAACTTTGAATT-3'; STAT3, sense, 5'-ATTCTACTGGAGTGCCGTAAC-3', anti-sense, 5'-ACAGGATGCGTAGGTTCTTG-3'; $\beta$-actin, sense, 5'-CATGTACGTTGCTATCCAGG-3', anti-sense, 5'-CTCCTTAATGTCACGCACGAT-3'. The parameters for PCR steps are as follows: predenaturation was at $95^{\circ} \mathrm{C}$ for 4 mins; denaturation was at $94{ }^{\circ} \mathrm{C}$ for $30 \mathrm{~s}$; annealing was at $58^{\circ} \mathrm{C}$ for $30 \mathrm{~s}$ and extension was at $72{ }^{\circ} \mathrm{C}$ for $30 \mathrm{~s}$ and 40 cycles. The expression levels of RNAs were calculated by $2^{-\Delta \Delta C t}$ method. All the assays were performed thrice.

\section{CCK-8}

The cells in normal group, hypoxia group (24 h), all other subgroups in hypoxia such as si-NC, si-GAS, si-HIF- $1 \alpha$, siGAS, si-GAS + si-HIF-1 $\alpha$ groups and AG490 group were cultured in 96-well plate with a quantity of $5 \times 10^{3}$ cells per well. Then, the cells were incubated at $37^{\circ} \mathrm{C}$ under $5 \%$ $\mathrm{CO}_{2}$. After incubation for $24 \mathrm{~h}, 10 \mu \mathrm{L}$ of CCK-8 solution (Beyotime, Shanghai, China) was added to each plate and then incubated for $1 \mathrm{~h}$. Then, the optical density (OD) values of the cells were measured at $450 \mathrm{~nm}$ wavelength using microplate reader (Thermo Fisher, USA). CCK-8 method was repeated thrice for all the groups.

\section{Cell apoptosis analysis by flow cytometry method}

Cells, from all the groups, were selected and seeded into 6 -well plate with $2 \times 10^{5}$ cells per well. Each group had three parallel complex holes. When the confluence of cells reached $80 \%$, the cells were collected and centrifuged at $1,200 \mathrm{rpm}$ for 5 mins. Then the cells were washed with PBS after which Annexin V-FITC/PE Apoptosis Detection Kit (Beyotime, Shanghai, China) was added to dying cells. Calibur flow cytometry (BDsciences, USA) was applied 
to detect the apoptosis of cells. The apoptosis rates were measured thrice for all the groups.

\section{Western blot}

H9C2 cells, from all the groups, were rinsed with PBS thrice and lysed with RIPA buffer (Beyotime, Shanghai, China) to extract total proteins. The concentration of these proteins were quantified using BCA method. After that, the total proteins were separated through SDS-PAGE and transferred to PVDF membranes (Beyotime, Shanghai, China). Thereafter, the PVDF membranes were blocked with 5\% skim milk powder at room temperature for $3 \mathrm{~h}$. Then the primary antibodies were added and incubated overnight at $4{ }^{\circ} \mathrm{C}$. In hypoxia subgroups i.e., si-NC, si-GAS + siNC, siGAS + si-HIF-1 $\alpha$ and AG490, phospho-JAK1, total JAK1, phospho-STAT3 and total STAT3 were detected by Western blot technique. The authors procured primary antibodies such as anti-IL-6 (1:1,000; ab229381, Abcam, Cambridge, UK), anti-IL-8 (1:1,000; ab252216), anti-p-JAK1 (1:1,000; PA5-37617, Thermo Fisher), anti-JAK1 (1:2,500, ab133666, Abcam), anti-p-STAT3 (1:2,000; ab76315, Abcam) and antiSTAT3 (1:2,000, ab68153, Abcam) with $\beta$-actin $(1: 2,000$; ab8226, Abcam) USA and maintained as internal reference. Then, the membranes were rinsed with QuickBlock ${ }^{\mathrm{TM}}$ Blocking Buffer (Beyotime, Shanghai, China) thrice and incubated with Goat Anti-Rabbit IgG H\&L (HRP) (1:2,000; ab205718) at room temperature for $2 \mathrm{~h}$. After that, the membranes were rinsed with PBST (Beyotime, Shanghai, China) thrice. BeyoECL Star solution was sprinkled onto the membranes ( $1 \mathrm{~mL} / 10 \mathrm{~cm}^{2}$, Beyotime, Shanghai, China) to cover it evenly. After 2 mins, the membranes were fixed by film clips and kept in dark chamber for $1 \mathrm{~min}$ for developing and fixing. The grey bands of proteins were scanned. Each group was evaluated thrice independently.

\section{Quantification of blot images}

The authors quantified the protein density of blot images using the software Image J, by determining the intensity of specific bands observed in western blot. The blot images were imported into software and contrast adjustments were made in such a way to obtain western blot images with clarity. Using rectangular selection tool, the area around each band was selected for analysis. The authors quantified the proteins p-JAK1, JAK1, p-STAT3, STAT3 using Image $\mathrm{J}$ and the ratios of p-JAK1 versus JAK1, p-STAT3 versus STAT3 are included in Figure S1.

\section{ELISA method}

Cell suspension in each group was collected to analyze IL- 6 and IL- 8 cytokines. Rat IL-6 ELISA kit was procured from SHHY Biotech (Shanghai, China) whereas rat IL-8 ELISA kit was bought from Huijia Biotech (Xiamen, Fujian, China). The authors strictly followed the instructions of both kits to estimate the concentrations of IL-6 and IL-8 cytokines. Each group was detected for IL-6 or IL-8 thrice.

\section{Statistical analysis}

Data is displayed as mean \pm SD values and was analyzed on SPSS 22.0 (IBM, USA). Each experiment was repeated for three times (biological replicates) and $t$-test was applied for comparison between two groups. One-way ANOVA was applied for groups more than two. Post-hoc analysis was conducted using Bonferroni's correction. The figures were drawn and plotted using GraphPad Prism8 (Graphpad Software, USA). $\mathrm{P}<0.05$ was considered to be statistically significant.

\section{Results}

Hypoxia-induced changes in cell morphology, cell viability, apoptosis and inflammation

The authors observed the cell morphology after H9C2 cells were exposed to hypoxic environment. The results showed that hypoxic treatment could bring changes in cell morphology such as polygonal cells and high refractive index (Figure 1A). Flow cytometry assays showed that the apoptosis rate of the cells got significantly elevated by hypoxia. Further, with increasing exposure to hypoxia, the apoptosis rate of the cells got increased (Figure 1B). CCK8 results inferred that the cells in hypoxia group had low cell viability (Figure $1 C$ ). Considering the huge difference between $24 \mathrm{~h}$ group and $0 \mathrm{~h}$ group, the authors selected $24 \mathrm{~h}$ hypoxia group for further assays. Inflammatory cytokines were evaluated by ELISA method. The results inferred that both IL-6 and IL-8 got significantly increased in hypoxiainduced H9C2 cells compared to normal H9C2 cells (Figure 1D).

\section{Upregulated GAS5 in bypoxia H9C2 cells and knockdown of GAS5 inbibiting apoptosis and inflammatory cytokines and promoting cell viability}

After transfection, RNAs were extracted from different groups. 
A
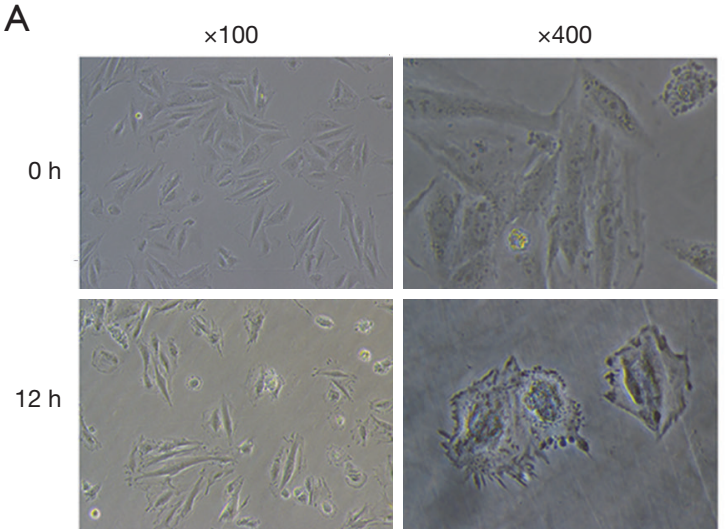

$24 \mathrm{~h}$
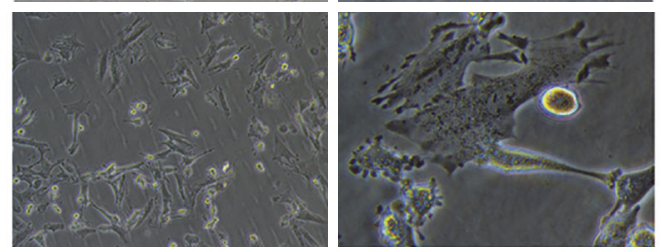

B
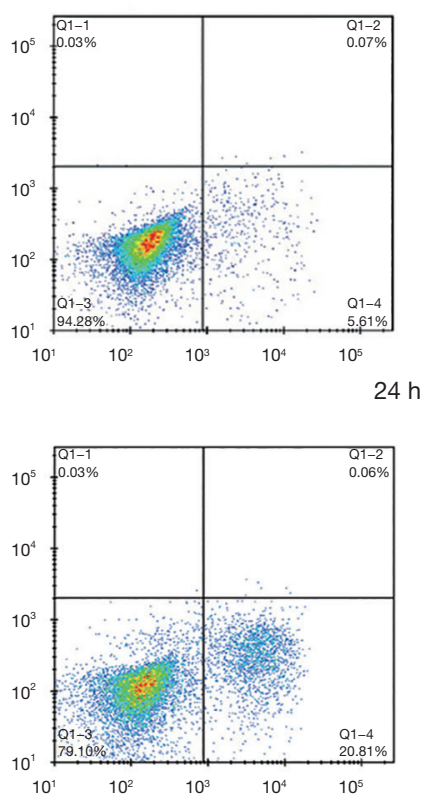

$\mathrm{Oh}$
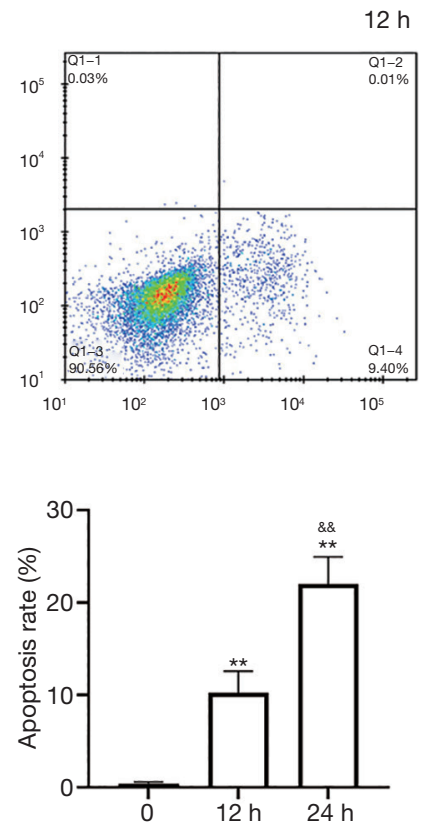

C
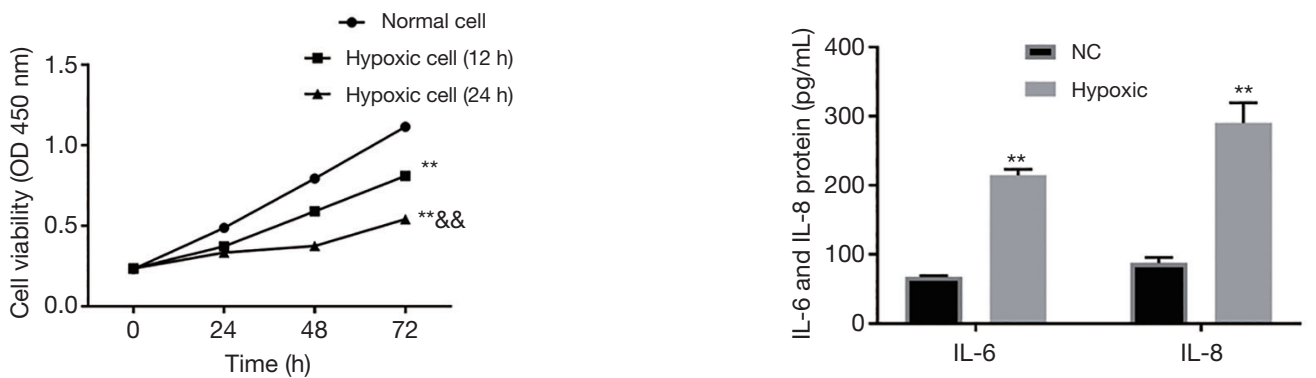

Figure 1 The establishment of the hypoxia-induced H9C2 cellular model. (A) Morphologies of H9C2 treated with hypoxia were observed and imaged under microscope in different magnifications $(\times 100$ and $\times 400)$ at 0,12 and $24 \mathrm{~h}$. Each time images were taken from six different angles and typical ones were selected for 0,12 and $24 \mathrm{~h} \times 100$ and $\times 400$ respectively. (B) Apoptosis rates were measured by Flow cytometry in cells with different hypoxia treatment (0, 12 and 24 h). (C) Cell viabilities were analyzed by CCK-8 assays in cells of normal group and hypoxia group (24 h). (D) ELISA methods were used to measure the concentrations of inflammatory cytokines IL-6 and IL- 8 in control and hypoxia groups. All assays were conducted for three independent times [ ${ }^{* *}, \mathrm{P}<0.05$, vs. normal cell group; ${ }^{\& \&}, \mathrm{P}<0.05$, hypoxia $(12 \mathrm{~h})$ group $v s$. hypoxia (24 h) group].

RNA electrophoresis images showed that the RNAs got successfully regulated in different groups (Figure 2A,B). RTqPCR found that GAS5 got significantly enhanced by hypoxia (Figure 2C). Furthermore, RT-qPCR results also verified the knockdown of GAS5 in si-GAS5 group (Figure 2D). Besides, ELISA results revealed the inhibition of IL- 6 and IL-8 protein expression (Figure 2E), indicating the alleviation of inflammation by downregulating GAS5 in hypoxic H9C2 cells
(Figure 2F,G,H).

\section{Upregulation of HIF-1a in hypoxic H9C2 cells and its knockdown inhibiting apoptosis and inflammatory cytokines and promoting cell viability}

Similar to GAS5, the expressions of HIF- $1 \alpha$ were also detected by RT-qPCR. There was a significant increase in 


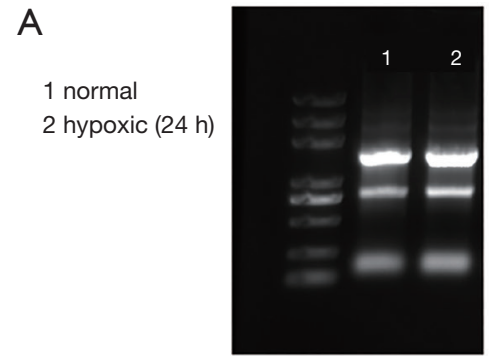

D

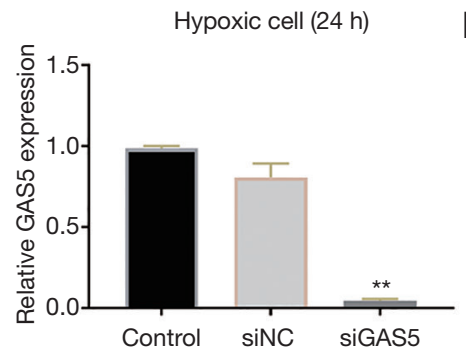

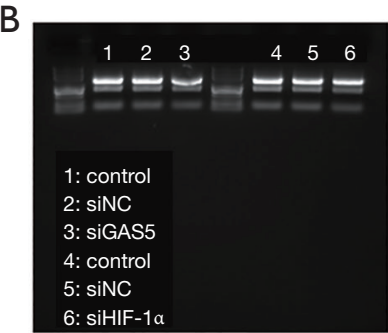

E

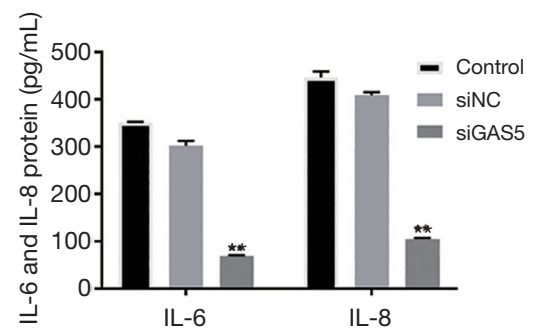

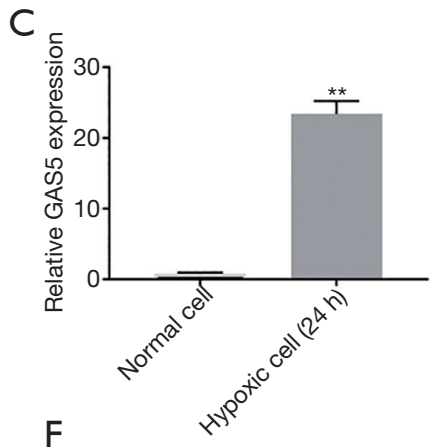

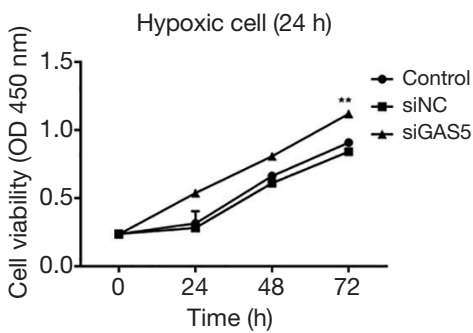

\section{G}
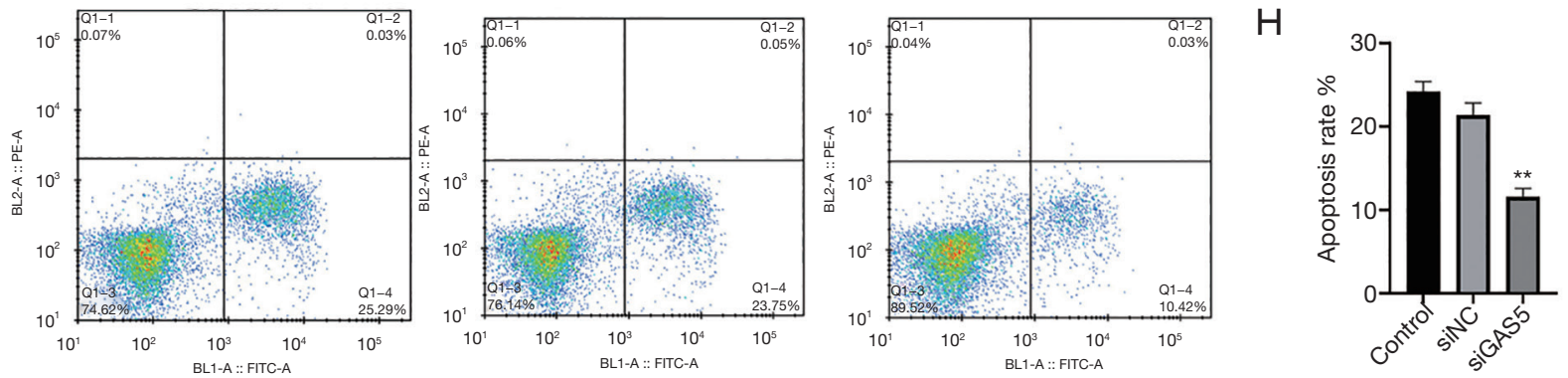

Figure 2 GAS5 was upregulated in hypoxia H9C2 cells and knockdown of GAS5 inhibited apoptosis and inflammatory cytokines and promoted cell viability. (A,B) electrophoretograms generated from cells after hypoxia treatment and transfections with si-NC, si-GAS5, siHIF-1 $\alpha$, etc. (C) RT-qPCR measured the relative RNA expressions of GAS5 in normal H9C2 and hypoxia-treated H9C2 group (**, P<0.05, $v s$. normal cell group). (D) The cells after exposure to hypoxic conditions for 24 hours were selected for cell transfection. The H9C2 cells in hypoxia group were transfected with si-NC and si-GAS5 with the hypoxia treated cells without transfection as a control $(* *, \mathrm{P}<0.05$, vs. control group). RT-qPCR measured the expressions of GAS5 in each group, namely, control, si-NC and si-GAS5 groups (**, $\mathrm{P}<0.05$, vs. control group). (E) ELISA methods assessed the inflammatory cytokines in control, si-NC and si-GAS5 groups (**, $\mathrm{P}<0.05$, vs. control group). (F) Cell viabilities were analyzed by CCK-8 assays in control, si-NC and si-GAS5 groups (**, $\mathrm{P}<0.05$, vs. control group). (G,H) Flow cytometry assays were used to detect the changes in apoptosis rates. All assays were conducted for three independent times $\left({ }^{* *}, \mathrm{P}<0.05\right.$, vs. control group). OD, optical density.

HIF-1 $\alpha$ levels during hypoxia treatment (Figure 3A). To further decrypt the role of HIF-1 $\alpha$ in hypoxia injury among H9C2 cells, HIF-1 $\alpha$ was silenced in H9C2 cells of hypoxia group. Both electrophoresis and RT-qPCR confirmed the knockdown of HIF-1 $\alpha$ in si-HIF-1 $\alpha$ group of H9C2 cells (Figures $2 B, 3 B$ ). Furthermore, the cell viabilities were evaluated after HIF-1 $\alpha$ was downregulated. The results indicate that the downregulation of HIF-1 $\alpha$ could promote cell viabilities (Figure 3C). Meanwhile, the concentrations of IL-6 and IL-8 were measured which concluded that low expression of HIF-1 $\alpha$ could inhibit inflammatory cytokines (Figure 3D). Moreover, flow cytometry assays found that the cell apoptosis rates got inhibited with the downregulation of GAS5 in hypoxic cells (Figure 3E). 
A

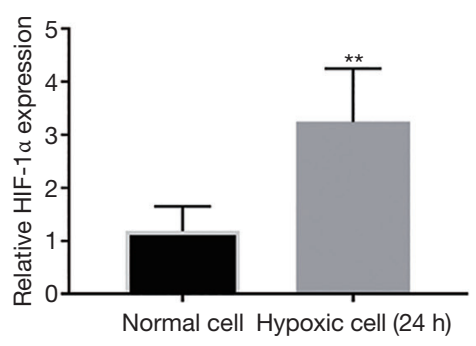

C

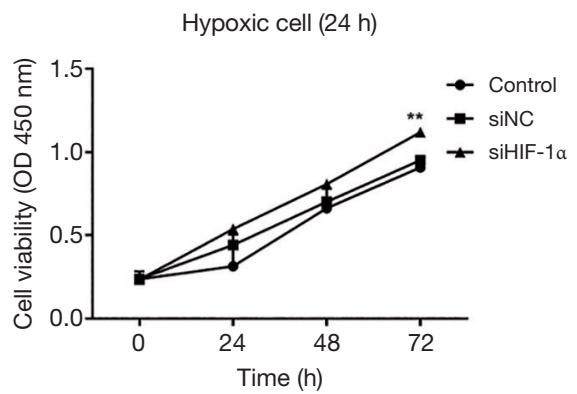

E
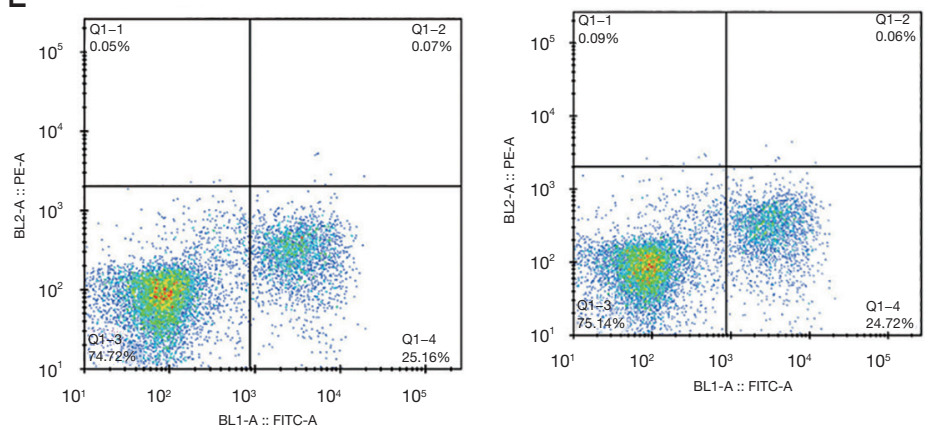

B

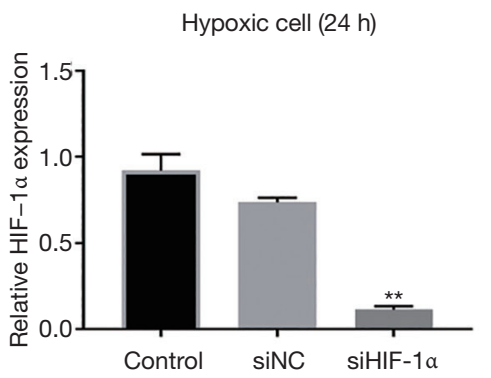

Figure $3 \mathrm{HIF}-1 \alpha$ was upregulated in hypoxia H9C2 cells and knockdown inhibited apoptosis and inflammatory cytokines and promoted cell viability. (A) HIF- $1 \alpha$ mRNA expressions in normal sand hypoxia group were checked through RT-qPCR (**, $\mathrm{P}<0.05$, vs. normal cell group). (B) The cells from hypoxia group were transfected with si-NC and si-HIF- $1 \alpha$ with the cells without transfection as a control (**, $\mathrm{P}<0.05, v s$. control group). RT-qPCR measured mRNA expressions of HIF- $1 \alpha$ in hypoxia control, si-NC and si-HIF-1 $\alpha$ groups ( ${ }^{*}, \mathrm{P}<0.05$, vs. control group). (C) Cell viabilities were measured through CCK-8 in hypoxia control, si-NC and si-HIF-1 $\alpha$ groups ${ }^{* *}, \mathrm{P}<0.05$, vs. control group). (D) IL-6 and IL-8 concentrations were quantified using ELISA methods ( ${ }^{* *}, \mathrm{P}<0.05$, vs. control group). (E) Apoptosis rates were detected in each group ${ }^{* *}, \mathrm{P}<0.05$, vs. control group). All assays were conducted for three independent times. OD, optical density.

\section{GAS5 and HIF-1a co-regulated bypoxic injury in H9C2 cells}

After detecting the functions of GAS5 and HIF-1 $\alpha$ independently, the interactions between GAS5 and HIF$1 \alpha$ were investigated in hypoxic H9C2 cells. CCK-8 results pointed out that the suppressed GAS5 could increase cell viability. Further, the inhibition of HIF-1 $\alpha$ could increase the viabilities of $\mathrm{H} 9 \mathrm{C} 2$ cells compared to si-GAS5 group (Figure 4A). Moreover, the inhibited GAS5 could suppress IL-6 and IL- 8 whereas the inhibition of HIF- $1 \alpha$ could add fuel to the fire (Figure 4B). Apoptosis assays showed that both si-GAS5 and si-HIF- $1 \alpha$ could co-function against hypoxia-induced apoptosis in $\mathrm{H} 9 \mathrm{C} 2$ cells better than si- 

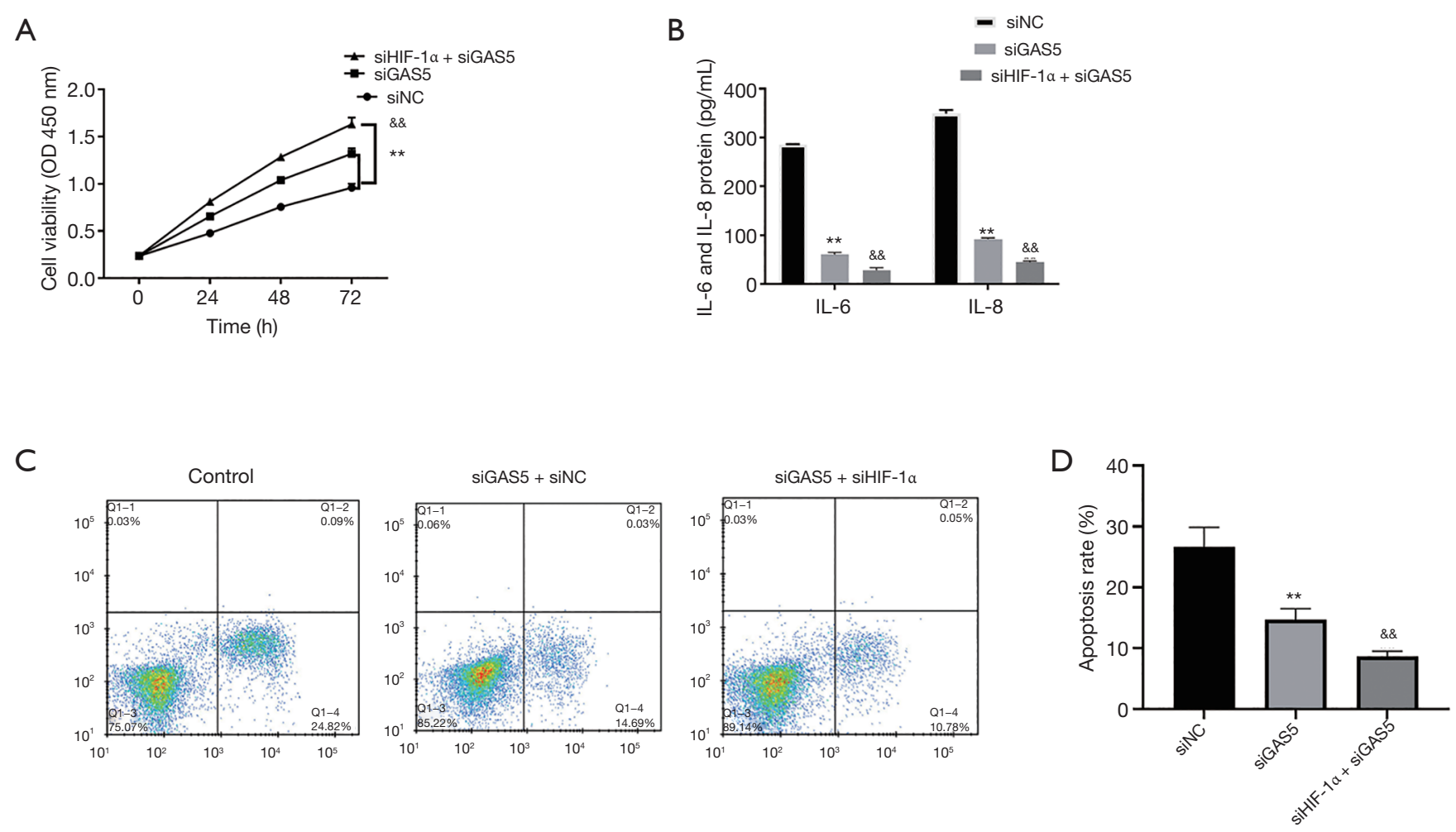

Figure 4 GAS5 and HIF-1 $\alpha$ co-regulated hypoxic injuries of H9C2 cells. The H9C2 cells from hypoxia group were transfected with si-NC, si-GAS, si-GAS + si-HIF-1 $\alpha$. (A) cell viabilities were checked using CCK-8. (B) Inflammatory cytokines were quantified by ELISA methods. (C,D) Flow cytometry was used to measure the apoptosis rates in each group. All assays were conducted for three independent times. **, $\mathrm{P}<0.05$ vs. si-NC group; ${ }^{\& \&}, \mathrm{P}<0.05$, vs. si-GAS group. OD, optical density.

GAS5 alone (Figure 4C,D).

\section{GAS5 and HIF-1 a co-regulated bypoxic injury in H9C2 cells via $\mathrm{FAK1/STAT3}$ signaling pathway}

RT-qPCR results inferred that mRNA expressions of JAK1 and STAT3 got significantly promoted in hypoxiainduced H9C2 cells compared to normal cells (Figure 5A). Furthermore, western blot technique found that the phosphorylation of JAK1 and STAT3 pathways got inhibited after GAS5 was inhibited. Both pathways got only mildly exhibited in the group co-transfected with si-HIF-1 $\alpha$ and si-GAS5 (Figure 5B; Figure S1A). To study the functions of JAK1 and STAT3 pathways in hypoxic injury of H9C2 cells, the signaling inhibitor AG-490 was applied to inactivate the signaling pathways. Western blot bands showed that AG-490 suppressed the phosphorylated levels of JAK1 and STAT3 (Figure 5C; Figure S1B). To investigate the impact of AG-490 on GAS5 and HIF-1 $\alpha$, RT-qPCR was used. It was found that the inactivation of JAK1/STAT3 signaling could deplete the expressions of GAS5 and HIF-1 $\alpha$ in H9C2 cells (Figure 5D). In addition, there was an increase observed in cell viability among AG-490 group cells compared to hypoxia control group (Figure 5E). ELISA method also showed that IL-6 and IL-8 got significantly inhibited by AG-490 (Figure 5F). Flow cytometry results showed that the apoptosis rates got inhibited by AG-490 (Figure 5G,H).

\section{Discussion}

Hypoxia is known to injure cardiac tissues and affect crucial cellular functions like proliferation and apoptosis (15). Proinflammatory cytokines, such as interleukin 6 (IL-6) and IL-8, are closely involved in the inflammation of various diseases. The depletion of IL-6 often results in protection from myocardial inflammation, cardiac dysfunction and fibrosis (16). Further, IL-6 and IL-8 are closely correlated with cardiac pathology especially cardiac failure, myocardial 
A

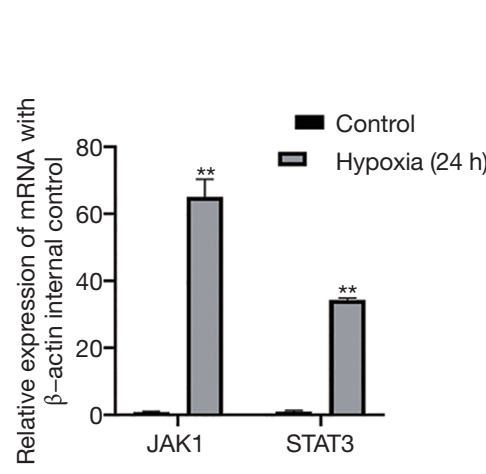

D

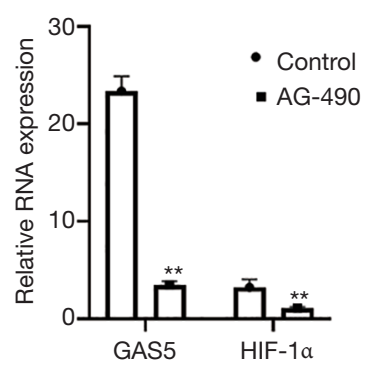

G

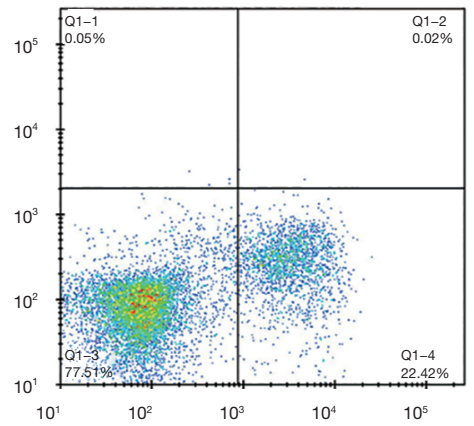

B

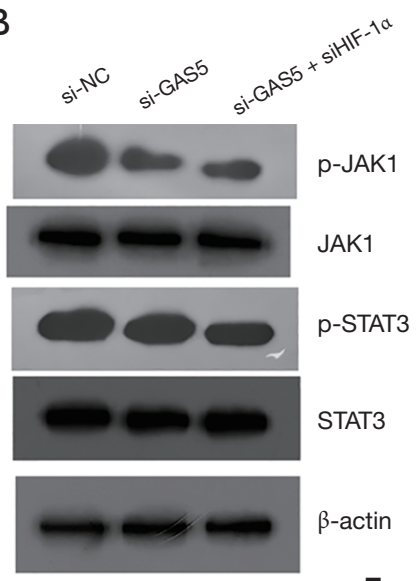

E

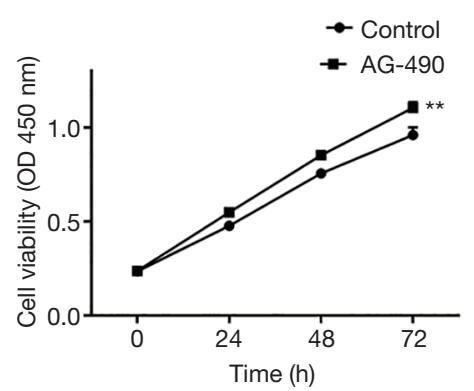

F
C

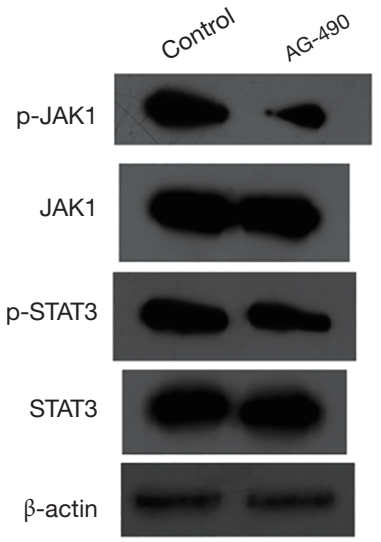

$F$

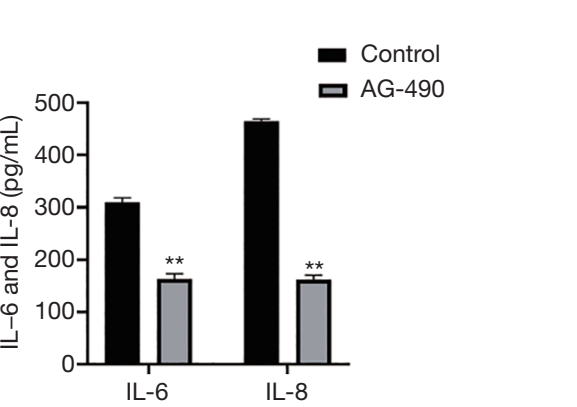

H

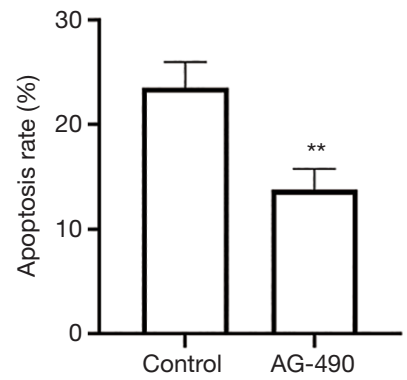

Figure 5 GAS5 and HIF-1 $\alpha$ regulated hypoxia injury in H9C2 cells via JAK1/STAT3 signaling. (A) mRNA expressions of JAK1 and STAT3 were measured by RT-qPCR in normal and hypoxia groups. (B) Western blot assays evaluated the p-JAK1/JAK1and p-STAT3/ STAT33 protein levels in si-NC, si-GAS and si-GAS + si-HIF-1 $\alpha$ groups. (C) The cells from hypoxia control group were treated with 10 nM AG-490 for 1 hour. Western blot assays evaluated the p-JAK1/JAK1 and p-STAT3/STAT3 protein levels in AG-490 group compared to hypoxia control group. (D) RT-qPCR also evaluated the relative expressions of GAS5 and HIF-1 $\alpha$ in AG-490 group compared to hypoxia control group. (E) Cell viabilities were measured through CCK-8. (F) ELISA methods quantified inflammatory cytokines in AG-490 group compared to hypoxia control group. $(\mathrm{G}, \mathrm{H})$ Flow cytometry analyzed apoptosis rates. All assays were conducted for three independent times. **, $\mathrm{P}<0.05$. OD, optical density.

infarction, and cardiomyopathies (17). Furthermore, hypoxia has been found to induce heavy expressions of cytokines in cardiomyocytes (18).

In this study, the authors exposed $\mathrm{H} 9 \mathrm{C} 2$ cells to hypoxic conditions for inducing hypoxic injury among cells in order to conduct in vitro analysis. To confirm the establishment of hypoxia $\mathrm{H} 9 \mathrm{C} 2$ model, the changes in cell morphology were observed under microscope at 0,12 and $24 \mathrm{~h}$ after exposure 
to hypoxic conditions. The increasingly-notable vacuolation signified that hypoxia-induced the death of H9C2 cells. Furthermore, functional assays also witnessed the occurrence of hypoxic injuries in hypoxia groups. It was also accompanied by reduced viabilities, activated inflammatory cytokines, and increased apoptosis rates in hypoxia groups compared to normal ones. Such characteristics of hypoxia injury in myocardial hypoxia cells have been reported earlier too $(19,20)$.

LncRNAs have been reported to have connections with hypoxia in tissues and cells. For instance, lncRNA-p21 can go aberrantly upregulated in anoxic condition and help in aggregating HIF-1 $\alpha$ among breast cancer cells (21). On the other hand, hypoxia could activate histone deacetylase, resulting in the deacetylation of promoters in IncRNALET to suppress its expression (22). LncRNA-LET had negative correlation with HIF-1 $\alpha$ in Ewing Sarcoma tumor growth (23).

LncRNA GAS5 was reported to play an important role in regulating cell cycle and apoptosis (24). In addition, lncRNA GAS5 acts as a tumor suppressor in different kinds of cancers such as bladder cancer (25), osteosarcoma (26), colorectal cancer, etc. (27).

In this research, the authors verified the protective role of downregulated GAS5 in H9C2 cells from hypoxia injury, by evaluating different parameters such as cell viability, apoptosis and inflammatory cytokines after the H9C2 cells were transfected with si-GAS5. The results were consistent with previous researches $(9,13)$. The functions of lncRNA GAS5 are being discovered in cardiovascular diseases in a gradual manner (28). Besides, in myocardial infarctioninduced rat and rat cardiocyte models, the knockdown of GAS5 promoted cell survival and inhibited apoptosis (13). It was disclosed earlier that Astragaloside IV protects the cardiomyocytes from hypoxia injury by downregulating lncRNA GAS5. This phenomenon suggested that a proper inhibitor of GAS5 might provide protective effect to cardiomyocytes from hypoxia (9). The suppression of lncRNA GAS5 was unveiled to protect rat cardiomyocytes H9C2 against hypoxic injury by targeting miR-222-3p (29). It is an established fact that HIF- $1 \alpha$ is accumulated in hypoxia-induced cells whereas the downregulation of HIF$1 \alpha$ inhibits cell apoptosis in cardiomyocytes after myocardial infarction (30). In the current study that assessed hypoxiainduced H9C2 cells, the expressions of HIF- $1 \alpha$ got significantly increased whereas the silencing of HIF- $1 \alpha$ also significantly contributed to cell viability and antagonized cell apoptosis and inflammatory cytokines. Likewise, we explored the role of HIF- $1 \alpha$ in $\mathrm{H} 9 \mathrm{C} 2$ against hypoxic injury. It was found that, similar to GAS5, the down-regulation of HIF- $1 \alpha$ could protect cells from hypoxia, thus promoting cell survival and inhibiting cell apoptosis and inflammatory cytokines.

The interactions between GAS5 and HIF-1 $\alpha$ were further evaluated in the current research. The results revealed that the inhibition of HIF- $1 \alpha$ could reinforce the functions of repressed GAS5, namely heavy protection for H9C2 cells against apoptosis and inflammation, promotion of cell survival which indicate that both GAS5 and HIF-1 $\alpha$ could co-regulate hypoxic injury of myocardial cells. In practice, some of the clinical trials, mostly cancer, occurred in HIF inhibitors (31). However, no clinical trial has been related to $\operatorname{lncRNA}$ and HIF-1 $\alpha$ as targets in cardiovascular diseases yet. This research provides new opportunities in the future possibly for a combined therapy of lncGAS5 into HIF- $1 \alpha$. Survivor activating factor enhancement (SAFE) pathway, activated in myocardial ischemia reperfusion injury, contains Janus kinase (JAK) and signal transducer and activator of transcription (STAT) (32). In mammals, JAK family consists of four members such as JAK1, JAK2, JAK3 and Tyk 2 and all of them are expressed in myocardium except JAK3 (33). STAT family is a group of underlying cytoplasmic transcription factors and its members are downstream targets which rely on tyrosine phosphorylation in JAK family $(34,35)$. All the members in STAT1 family i.e., STAT1, STAT2, STAT3, STAT4, STAT5A, STAT5B and STAT6, are expressed in heart tissues (36). Studies have concluded that the activation of STAT1/3 has a relationship with apoptosis of neonatal rat cardiomyocytes stimulated by ischemia (37). AG490 is a JAK inhibitor which mainly blocks JAK1 and JAK2. This action primarily results in the inhibition of STAT3 which was exposed to alleviate myocardial infarction after ischemia reperfusion (38-40).

In this study, hypoxic cells exhibited high mRNA expressions of JAK1 and STAT3 compared to normal H9C2. Moreover, JAK1 and STAT3 had positive correlation with GAS5 and got inhibited more significantly in the combined group of si-GAS5 and si-HIF-1 $\alpha$ than si-GAS5 group. After AG490 was used, JAK1 and STAT3 got inhibited effectively and accordingly the RNA expressions of GAS5 and HIF- $1 \alpha$. This phenomenon in turn indicates that both GAS5 and HIF- $1 \alpha$ could be modulated by JAK1/STAT3 signaling. Meanwhile, cell viability got promoted and apoptosis and inflammation got alleviated by AG-490. This indicates that the inactivation of JAK1/STAT3 signaling pathway ameliorated the hypoxic injury of $\mathrm{H} 9 \mathrm{C} 2$ cells. 


\section{Conclusions}

In this study, both lncRNA GAS 5 and HIF- $1 \alpha$ got heavily expressed in hypoxia-treated myocardial cells. The knockdown of GAS5 was able to synergize with the inhibition of HIF- $1 \alpha$ to protect myocardial cells from hypoxic injury. JAK1/STAT3 pathway got activated in hypoxia cells which could be inactivated by the inhibition of GAS5 5 and HIF- $1 \alpha$. Besides, the application of AG490, an antagonist of JAK1/STAT3 pathway resulted in the inhibition of GAS 5 and HIF- $1 \alpha$ in cells, leading to alleviation of hypoxic injury. Therefore, the authors conclude that si-GAS5 got synergized with si-HIF-1 $\alpha$ to alleviate hypoxic injury in myocardial H9C2 cells via JAK1/ STAT3 pathway. However, our findings in this research are only based on in vitro findings which calls for more intensive and in-depth investigations in both animals and in clinical trials.

\section{Acknowledgments}

Funding: None.

\section{Footnote}

Reporting Checklist: The authors have completed the MDAR reporting checklist. Available at http://dx.doi.org/10.21037/ cdt-20-773

Data Sharing Statement: Available at http://dx.doi. org/10.21037/cdt-20-773

Peer Review File: Available at http://dx.doi.org/10.21037/ cdt-20-773

Conflicts of Interest: All authors have completed the ICMJE uniform disclosure form (available at http://dx.doi. org/10.21037/cdt-20-773). The authors have no conflicts of interest to declare.

Ethical Statement: The authors are accountable for all aspects of the work in ensuring that questions related to the accuracy or integrity of any part of the work are appropriately investigated and resolved. All the experiments were performed in this study in compliance with the guidelines of Institutional Ethical Review Board (IEC), The First Affiliated Hospital, Jinzhou Medical University, Jinzhou City, Liaoning Province, China.
Open Access Statement: This is an Open Access article distributed in accordance with the Creative Commons Attribution-NonCommercial-NoDerivs 4.0 International License (CC BY-NC-ND 4.0), which permits the noncommercial replication and distribution of the article with the strict proviso that no changes or edits are made and the original work is properly cited (including links to both the formal publication through the relevant DOI and the license). See: https://creativecommons.org/licenses/by-nc-nd/4.0/.

\section{References}

1. Kang PM, Haunstetter A, Aoki H, et al. Morphological and molecular characterization of adult cardiomyocyte apoptosis during hypoxia and reoxygenation. Circ Res 2000;87:118-25.

2. Bouitbir J, Alshaikhali A, Panajatovic M, et al. Mechanisms of cardiotoxicity associated with tyrosine kinase inhibitors in H9c2 cells and mice. Eur Cardiol 2020;15:e33.

3. Parente V, Balasso S, Pompilio G, et al. Hypoxia/ reoxygenation cardiac injury and regeneration in zebrafish adult heart. PLoS One 2013;8:e53748.

4. Schmitt AM, Chang HY. Long noncoding RNAs in cancer pathways. Cancer Cell 2016;29:452-63.

5. Yan Y, Song D, Song X, et al. The role of lncRNA MALAT1 in cardiovascular disease. IUBMB Life 2020;72:334-42.

6. Yang X, Xie Z, Lei X, et al. Long non-coding RNA GAS5 in human cancer. Oncol Lett 2020;20:2587-94.

7. Peng H, Luo Y, Ying Y. lncRNA XIST attenuates hypoxiainduced $\mathrm{H} 9 \mathrm{c} 2$ cardiomyocyte injury by targeting the miR122-5p/FOXP2 axis. Mol Cell Probes 2020;50:101500.

8. Liu SD, Meng WX, Xu L, et al. GAS5 promotes myocardial apoptosis in myocardial ischemia-reperfusion injury via upregulating LAS1 expression. Eur Rev Med Pharmacol Sci 2018;22:8447-53.

9. Du J, Liu J, Zhen J, et al. Astragaloside IV protects cardiomyocytes from hypoxia-induced injury by downregulation of lncRNA GAS5. Biomed Pharmacother 2019;116:109028.

10. Feng CC, Lin CC, Lai YP, et al. Hypoxia suppresses myocardial survival pathway through HIF-1 $\alpha$-IGFBP3-dependent signaling and enhances cardiomyocyte autophagic and apoptotic effects mainly via FoxO3ainduced BNIP3 expression. Growth Factors 2016;34:73-86.

11. Pu J, Zhu S, Zhou D, et al. Propofol Alleviates Apoptosis 
Induced by Chronic High Glucose Exposure via Regulation of HIF-1 in H9c2 Cells. Oxid Med Cell Longev 2019;2019:4824035.

12. Shi YN, Zhang XQ, Hu ZY, et al. Genistein protects $H 9 c 2$ cardiomyocytes against chemical hypoxia-induced injury via inhibition of apoptosis. Pharmacology 2019;103:282-90.

13. Zhou XH, Chai HX, Bai M, et al. LncRNA-GAS5 regulates PDCD4 expression and mediates myocardial infarction-induced cardiomyocytes apoptosis via targeting MiR-21. Cell Cycle 2020;19:1363-77.

14. Zhang Y, Liu D, Hu H, et al. HIF-1 $\alpha$ /BNIP3 signaling pathway-induced-autophagy plays protective role during myocardial ischemia-reperfusion injury. Biomed Pharmacother 2019;120:109464.

15. Semenza GL. Hypoxia-inducible factors in physiology and medicine. Cell 2012;148:399-408.

16. González GE, Rhaleb NE, D'ambrosio MA, et al. Deletion of interleukin-6 prevents cardiac inflammation, fibrosis and dysfunction without affecting blood pressure in angiotensin II-high salt-induced hypertension. J Hypertens 2015;33:144-52.

17. Bartekova M, Radosinska J, Jelemensky M, et al. Role of cytokines and inflammation in heart function during health and disease. Heart Fail Rev 2018;23:733-58.

18. Wu J, Stefaniak J, Hafner C, et al. Intermittent hypoxia causes inflammation and injury to human adult cardiac myocytes. Anesth Analg 2016;122:373-80.

19. Wei Q, Zhou HY, Shi XD, et al. Long noncoding RNA NEAT1 promotes myocardiocyte apoptosis and suppresses proliferation through regulation of miR-129-5p. J Cardiovasc Pharmacol 2019;74:535-41.

20. Kuang Y, Huang YS. Effect of the microtubule depolymerization on mitochondria damage in rat myocardiocytes early after hypoxia. Zhonghua Shao Shang Za Zhi 2007;23:288-91.

21. Yang F, Zhang H, Mei Y, et al. Reciprocal regulation of HIF-1alpha and lincRNA-p21 modulates the Warburg effect. Mol Cell 2014;53:88-100.

22. Yang F, Huo XS, Yuan SX, et al. Repression of the long noncoding RNA-LET by histone deacetylase 3 contributes to hypoxia-mediated metastasis. Mol Cell 2013;49:1083-96.

23. Hameiri-Grossman M, Porat-Klein A, Yaniv I, et al. The association between let-7, RAS and HIF-1alpha in Ewing Sarcoma tumor growth. Oncotarget 2015;6:33834-48.

24. Li Y, Li Y, Huang S, et al. Long non-coding RNA growth arrest specific transcript 5 acts as a tumour suppressor in colorectal cancer by inhibiting interleukin-10 and vascular endothelial growth factor expression. Oncotarget 2017;8:13690-702.

25. Wang M, Guo C, Wang L, et al. Long noncoding RNA GAS5 promotes bladder cancer cells apoptosis through inhibiting EZH2 transcription. Cell Death Dis 2018;9:238.

26. Wang Y, Kong D. LncRNA GAS5 represses osteosarcoma cells growth and metastasis via sponging MiR-203a. Cell Physiol Biochem 2018;45:844-55.

27. Li J, Wang Y, Zhang CG, et al. Effect of long non-coding RNA Gas5 on proliferation, migration, invasion and apoptosis of colorectal cancer HT-29 cell line. Cancer Cell Int 2018;18:4. Erratum in: Cancer Cell Int 2018;18:17.

28. Chen L, Yang W, Guo Y, et al. Exosomal lncRNA GAS5 regulates the apoptosis of macrophages and vascular endothelial cells in atherosclerosis. PLoS One 2017;12:e0185406.

29. Du J, Yang ST, Liu J, et al. Silence of lncRNA GAS5 protects cardiomyocytes H9c2 against hypoxic injury via sponging miR-142-5p. Mol Cells 2019;42:397-405.

30. Wu L, Chen Y, Chen Y, et al. Effect of HIF-1 $\alpha / \mathrm{miR}-10 b-$ $5 \mathrm{p} / \mathrm{PTEN}$ on hypoxia-induced cardiomyocyte apoptosis. J Am Heart Assoc 2019;8:e011948.

31. Fallah J, Rini BI. HIF inhibitors: status of current clinical development. Curr Oncol Rep 2019;21:6.

32. Lecour S. Multiple protective pathways against reperfusion injury: a SAFE path without Aktion? J Mol Cell Cardiol 2009;46:607-9.

33. Aringer M, Cheng A, Nelson JW, et al. Janus kinases and their role in growth and disease. Life Sci 1999;64:2173-86.

34. Ihle JN. The Stat family in cytokine signaling. Curr Opin Cell Biol 2001;13:211-7.

35. Horvath CM. STAT proteins and transcriptional responses to extracellular signals. Trends Biochem Sci 2000;25:496-502.

36. Xuan YT, Guo Y, Han H, et al. An essential role of the JAK-STAT pathway in ischemic preconditioning. Proc Natl Acad Sci U S A 2001;98:9050-5.

37. McWhinney CD, Hunt RA, Conrad KM, et al. The type I angiotensin II receptor couples to Stat1 and Stat3 activation through Jak2 kinase in neonatal rat cardiac myocytes. J Mol Cell Cardiol 1997;29:2513-24.

38. Gorina R, Petegnief V, Chamorro Á, et al. AG490 prevents cell death after exposure of rat astrocytes to hydrogen peroxide or proinflammatory cytokines: involvement of the Jak2/STAT pathway. J Neurochem 2005;92:505-18.

39. Eriksen KW, Kaltoft K, Mikkelsen G, et al. Constitutive STAT3-activation in Sezary syndrome: tyrphostin 
AG490 inhibits STAT3-activation, interleukin-2 receptor expression and growth of leukemic Sezary cells. Leukemia 2001;15:787-93.

Cite this article as: Li Y, Song B, Liu J, Li Y, Wang J, Liu N, Cui W. The interplay between HIF- $1 \alpha$ and long noncoding GAS5 regulates the JAK1/STAT3 signalling pathway in hypoxia-induced injury in myocardial cells. Cardiovasc Diagn Ther 2021;11(2):422-434. doi: 10.21037/cdt-20-773
40. Mascareno E, El-Shafei M, Maulik N, et al. JAK/STAT signaling is associated with cardiac dysfunction during ischemia and reperfusion. Circulation 2001;104:325-9. 
A

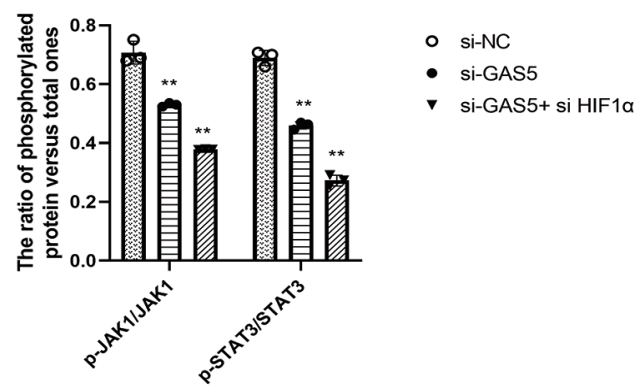

B

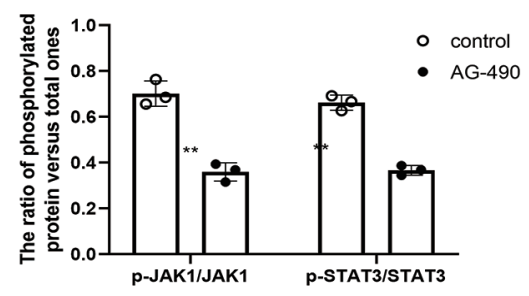

Figure S1 Quantification of protein density of blot images were done using software Image J by determining the intensity of the specific bands in the western blot. The intensities of the bands are displayed and the relative concentration of protein in the sample is quantified. Results are mean values of three independent experiments. $(\mathrm{A}, \mathrm{B})$ The western blot quantitation (Image J) of the ratios of p-JAK1/JAK1 and p-STAT3/STAT3 in AG-490 group compared to hypoxia control group. 